TERRA. Revista de Desarrollo Local e-ISSN: 2386-9968

Número 6 (2020), 370-383

DOI 10.7203/terra.6.16997

IIDL - Instituto Interuniversitario de Desarrollo Local

\title{
Reseña. El desarrollo del análisis de redes sociales: un estudio de sociología de la ciencia
}

\section{Rafael Mesa Manzano}

Graduado en Geografía y Medio Ambiente, y Máster en Técnicas para la Gestión del Medio Ambiente y del Territorio (Universidad de Valencia, España)

Rafael.Mesa@uv.es

https://orcid.org/0000-0001-9525-6700

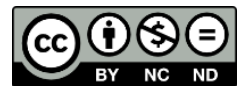

Esta obra se distribuye con la licencia Creative Commons

Reconocimiento-NoComercial-SinObraDerivada 4.0 Internacional 


\section{SECCIÓN RESEÑAS}

\section{Reseña. El desarrollo del análisis de redes sociales: un estudio de sociología de la ciencia}

Resumen. El desarrollo del análisis de redes sociales ha tenido una trayectoria fragmentada a lo largo de la historia reciente. Las contribuciones al análisis de redes no han evolucionado de manera conjunta, sino que diversas escuelas o tradiciones han realizado sus progresos por separado entre periodos con mayor y menor actividad. Su evolución ha estado marcada por una progresiva especialización donde han intervenido multitud de disciplinas. Así, las cuatro características que definen el análisis de redes sociales son: el enfoque estructural, el sistemático, el empleo de grafos y el uso de modelos matemáticos y computacionales. A día de hoy, el análisis de redes sociales es una disciplina madura que cuenta con numerosas publicaciones desde distintos campos de aplicación.

Palabras clave: Análisis de redes sociales, enfoque estructural, sociometría.

Recibido: 06 de abril de 2020

Devuelto para revisión: -

Aceptado: 06 de abril de 2020

Referencia / Citation:

Mesa, R. (2020). Reseña. El desarrollo del análisis de redes sociales: un estudio de sociología de la ciencia. TERRA. Revista de Desarrollo Local, (6), 370-383. DOI 10.7203/terra.6.16997 


\section{EL DESARROLLO DEL ANÁLISIS DE REDES SOCIALES: UN ESTUDIO DE SOCIOLOGÍA DE LA CIENCIA}

Palibrio, 2012. 208 páginas.

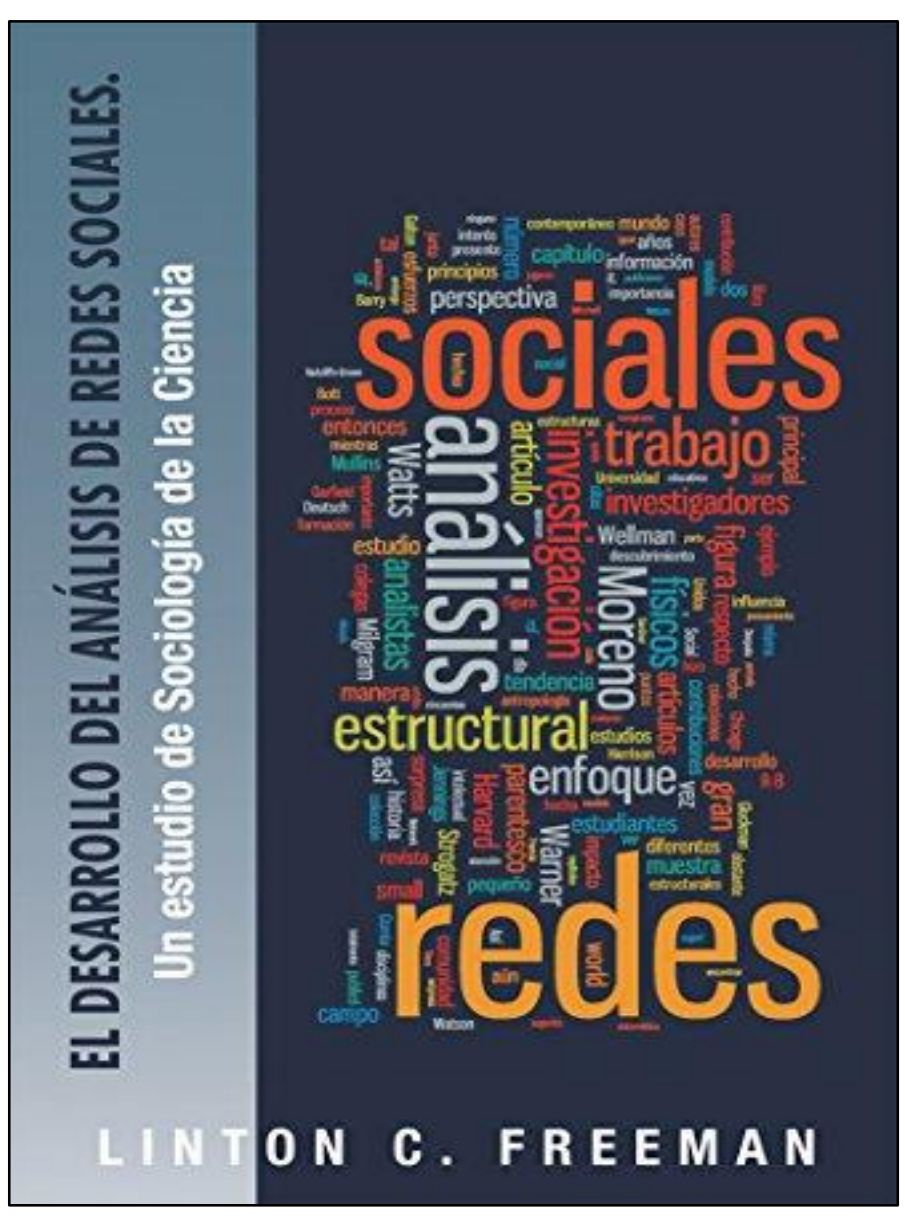

En la actualidad, el progreso que ha tenido la ciencia de redes es claro, considerando que su aplicación traspasa disciplinas, desde la Antropología, donde se dieron los ejemplos más tempranos en el trabajo sistemático, a la Sociología, la Psicología, Biología, Geografía, Física y Matemáticas, entre otras, con importantes contribuciones. En el momento de la publicación del libro en el año 2004 bajo el título original "The Development of Social Network Analysis: A Study in the Sociology of Science", el número de artículos de análisis de redes sociales listados en Sociological Abstracts, desde el 1974 hasta el 1999, no había dejado de aumentar (p. 172). Desde hace dos décadas hasta la actualidad es bien sabido que el análisis de redes sociales es una disciplina madura.

Linton Freeman, autor de libro, fue un destacado sociólogo estructuralista y referente en el campo del análisis de redes sociales con numerosas aportaciones, desde la enseñanza a la producción de artículos y libros. Es conocido por ser el primer autor en desarrollar la intermediación como medida de centralidad (Freeman, 1978), además de ser el fundador de la revista Social Networks en 1979. Luego, el papel de Freeman, puede decirse que ha sido imprescindible y fundamental.

Asimismo, en este libro, el autor subraya que no pretende ser una obra de la historia del análisis de redes sociales, aunque, no obstante, sin quererlo, ciertamente lo consigue. En la introducción (Capítulo 1), Freeman señala las cuatro características fundamentales que definen el análisis de redes sociales, y que han tenido lugar recientemente (p. 3):

1. El análisis de redes sociales parte de la intuición estructural de la existencia de lazos que ligan a actores sociales.

2. Está basado en información empírica sistemática.

3. Hacia amplio uso de imágenes gráficas, y

4. Utiliza modelos matemáticos o computacionales. 
En este libro, Freeman realiza una exhaustiva revisión literaria y trabajo de campo cuyo recorrido abarca una considerable línea temporal, desde "La prehistoria de las ideas y de la práctica de las redes sociales" (Capítulo 2), pasando, en primer lugar, por los aportes de Jacob Moreno y Helen Jennings y, en segundo lugar, por las contribuciones de Harrison White (1963) (Capítulos 3 y 4, respectivamente). Continua con el análisis de redes sociales durante el oscurantismo de los años cuarenta, cincuenta y sesenta, un periodo al que el autor se refiere como "las tinieblas" del análisis de redes sociales debido a una baja actividad, pese a que nunca estuvo totalmente detenido (Capítulos 5, 6 y 7). En los tres últimos capítulos encontramos la evolución reciente del análisis de redes sociales bajo el título "Renacimiento de Harvard" (Capítulo 8), "El análisis de redes sociales se organiza" (Capítulo 9) y "Un sumario y varias sorpresas" (Capítulo 10). En estos tres capítulos se realiza un repaso de las diversas aportaciones que han contribuido al desarrollo del análisis de redes sociales, como son, el prominente papel de la Universidad de Harvard, el desarrollo de programas computacionales y conferencias, la creación de una revista específica de análisis de redes sociales, la importancia de los congresos como medio integrador y vehículo para hacer crecer sostener la comunidad, y el papel destacado de la Universidad de California en Irvine. De esta manera, en el último capítulo encontramos una síntesis del proceso de investigación que dio lugar al libro "El desarrollo del análisis de redes sociales: un estudio de sociología de la ciencia", así como diversos descubrimientos y opiniones personales por parte del autor que tuvieron lugar al realizar entrevistas a actores relevantes en el análisis de redes sociales.

\section{PREHISTORIA DEL ANÁLISIS DE REDES SOCIALES. ANTERIOR A LOS AÑOS 30 (CAPÍTULO 2)}

En este capítulo el autor señala los cuatros rasgos característicos del análisis de redes sociales mencionados anteriormente. Además, realiza un repaso sobre autores que normalmente no son citados y que introdujeron innovaciones en el análisis de redes sociales. Por ejemplo, entre los trabajos más tempranos bajo un enfoque sistemático se sitúa el del naturalista Pierre Huber, el cual se dedicó al estudio de las abejas y hormigas (Huber, 1810; Huber \& Bonnet, 1792), o las contribuciones de Auguste Comte (Comte \& Martineau, 1853), donde se pueden encontrar las primeras afirmaciones explícitas sobre redes y quien, además, acuñó el término de sociología. Comte adoptó un punto de vista estructural refiriéndose a la sociedad en términos de conexiones entre actores, mostrando que la sociedad estaba interconectada formando parte de un sistema social. Este tipo de análisis estructural fue adoptado por destacados científicos durante el siglo diecinueve y veinte como, por ejemplo, Gustave LeBón (1897-1995) (LeBón, 1895) que adoptó un enfoque estructural diferente estudiando el comportamiento de masas en relación al flujo de información entre individuos. Sin embargo, la contribución más explícita fue la de Georg Simmel (1908-1971) (Simmel \& Levine, 1971), afirmando que "la sociedad existe ahí en donde un número de individuos interactúan". Por otro lado, el abogado y antropólogo Lewis Henry Morgan en 1851 realizó un estudio de parentesco sobre las tribus norteamericanas (Morgan, 2018), mientras que, cien años después del mencionado Huber, John Atkinson Hobson (1894-1954) desarrolló un enfoque bimodal donde se reflejaban la relación entre individuos y organizaciones. Desde la Psicología se realizaron importantes contribuciones escasamente reconocidas durante los años veinte, época la cual se destinaron recursos para estudios sobre las relaciones interpersonales en la infancia. Durante esta época se realizaron estudios sobre la homofilia en niños de edad escolar aplicando un enfoque estructural: 1) preguntando a los niños a quién invitarían a 
una fiesta (Almack, 1922); 2) preguntando con quién jugaría a la hora del recreo (Wellman, 1926); 3) Helen Bott (1928), redefinió el método anterior bajo un enfoque etnográfico, recolectando información detalla de interacciones entre niños (esta autora es escasamente citada en la literatura y, según Freeman, realizó significativas aportaciones siendo pionera en métodos de recolección y presentación de datos); 4) en 1933 Elizabeth Hagman y Stoddard en 1933 realizaron estudios donde se combinan entrevistas bajo métodos observacionales.

La representación gráfica es una parte transcendental dentro del análisis de redes sociales. Entre los primeros estudios se hallan los lazos de parentesco más básicos como los árboles genealógicos. Científicos como el mencionado Lewis Henry Morgan introdujo gráficos donde se representaban la relación de parentesco de la antigua Roma. Por otro lado, en el siglo diecinueve, Macfarlane (1883) desarrolló un modelo visual de parentesco mientras que Hobson (1894) representó su red bimodal por medio de grafos.

Finalmente, en este capítulo Freeman señala que Georg Simmel ha sido reconocido como una parte importante dentro del enfoque de redes, mientras que otros autores apenas han sido mencionados.

\section{EL NACIMIENTO DEL ANÁLISIS DE REDES SOCIALES}

\subsection{La sociometría (Capítulo 3)}

El capítulo 3, "El nacimiento del análisis de redes sociales", está dedicado a la biografía de Jacob Moreno, reconocido como el principal motor de la sociometría (Moreno, 1937, 1953). La vida de Moreno está rodeada de un halo místico y se le describe como un personaje "enigmático, brillante, creativo, divertido y con una energía inagotable". Moreno comenzó a trabajar con diagramas sociométricos sobre beneficios terapéuticos. Viajó desde Austria a Estados Unidos donde conoció a Helen Hal Jennings, una estudiante de postgrado de la Universidad de Columbia. Jennings jugó un papel muy importante en los trabajos de Moreno (Moreno \& Jennings, 1938, 1960). Durante este periodo, buena parte de las aportaciones de la perspectiva estructural venían del campo de Psicología, donde se empleaban técnicas sociométricas para estudiar los sentimientos de las personas. Durante este periodo, Moreno fundó dos revistas, la primera, Sociometric Review, la cual fue cerrada para un año más tarde crear la revista Sociometry. Otro actor destacado en la trayectoria de Moreno fue Paul Lazarsfeld, sociólogo y matemático de la Universidad de Columbia. Lazarsfeld trabajó el análisis de probabilidades.

Las aportaciones de Moreno se han visto empañadas por su carácter, sin embargo, como señala Freeman citando a Marineau (1989, p. 121), biógrafo de Moreno: "muy pocos estudiantes de psicología y sociología social de la actualidad podrían jamás sospechar del impacto que Moreno tuvo en este campo de investigación hace más de cincuenta años".

\subsection{El primer atisbo en Harvard (Capítulo 4)}

Desde Harvard, a finales de los años veinte, se comenzaron a estudiar las estructuras de red por parte de William Lloyd Warner. Warner trabajó recopilando información etnográfica y de parentesco con la ayuda de Radcliffe-Brown. Entre sus estudios se encuentra el llamado "Yankee City" de 1963 (Warner, 1975), el cual se centraba en la estratificación empleando el marco teórico de Durkheim. Según describe Freeman, 
Warner trabajaba con un enfoque estructural con ideas sofisticadas para la época, donde se aportaron gráficos e hipergráficas sobre la estructura general y cliques. Las investigaciones de Warner continuaron con el proyecto "Deep South" (A. Davis, Gardner, Gardner, \& Warner, 1941), en el cual se estudiaba el impacto de las diferencias raciales. En este estudio se emplearon gráficos y recolección sistemática de datos sin aplicar modelos matemáticos, no obstante, quedó reflejado el dominio de la perspectiva estructural de Warner.

Durante un seminario permanente en Harvard organizado por el propio Warner participaron investigadores de la Western Electric como Elton Mayo, Whitehead, Roethlisberger y Lawrence Joseph Henderson. Para Henderson, los estudios de Pareto sirvieron de inspiración. Henderson estableció vínculos con Homans y este último tuvo acceso a fuentes de conocimiento que en aquel momento estaban a la vanguardia de la psicología. Homans publicó en 1950 un libro titulado "The Human Group" donde aplicaron fundamentos estructurales (Homans, 2013).

Eliot Chapple y Conrad Arensberg promovieron un marco teórico para la recolección y análisis de datos estructurales de manera sistemática y definieron la variable de interacción (Chapple \& Arensberg, 1940). Ambos investigadores se enfrentaban al problema de la generalización de la información. Por lo tanto, con la ayuda de otro matemático de Harvard, Willard Quine, establecieron un modelo algebraico derivado de un modelo de parentesco. Al disponer de este modelo ya podrían completar las cuatro características que caracterizan el análisis de redes sociales.

En resumen, Homans cuenta con algunas menciones ocasionales, por el contrario, los estudios de Roethlisberger y Dickson (1939), Davis y Gardner and Gardner (1941) tuvieron mayor aceptación en la comunidad científica. Tras los aportes de Moreno y el de los investigadores de Harvard, el análisis de redes sociales no tuvo centros importantes de investigación, pese ello, Freeman destaca que durante un tiempo existieron contribuciones que permitieron que el enfoque estructural continuara dominando.

\section{LA ÉPOCA DEL OSCURANTISMO}

\subsection{Los años cuarenta (Capítulo 5)}

En los años cuarenta, tanto las influencias de Moreno y Jennings, como los aportes de Harvard se habían disipado. El enfoque de redes había dejado de ser considerado desde el punto de vista teórico y práctico. Aun así, algunas escuelas continuaron aplicándolo.

En primer lugar, el MIT (Massachusetts Institute of Technology) donde Kurt Lewin fundó el Centro de Investigación en Dinámica de Grupos (más tarde desplazado a la Universidad de Michigan) junto con Doc Cartwright, Leon Festinger, Ronald Lippitt, Marian Radke y Alex Bavelas. Bavelas (1948) realizó aportes estudiando la importancia del grado de centralización de los individuos y fundó el Laboratorio de Redes Grupales en el MIT con la ayuda de Festinger. A esto se le sumaron estudiantes e investigadores como Albert Perry y Duncan Luce. Juntos prepararon un artículo donde de manera formal aparece la definición de "clique" (Luce \& Perry, 1949). El grupo del MIT fue otro grupo que trabajó con las cuatro características que definen el análisis de redes sociales. Las investigaciones continuaron y, tanto Cartwright como Festigner, se vieron con la necesidad de mejorar las técnicas para analizar los patrones de comportamiento a través de fundamentos matemáticos. 
En segundo lugar, a mediados de los cuarentas algunos de los seguidores de Moreno continuaron con la sociometría. El sociólogo rural Charles P. Llomis, director del Departamento de Sociología y Antropología del Colegio Estatal de Michigan, promovió entre sus estudiantes el análisis estructural y se preocupó por la importancia de las matemáticas. Loomis realizó estudios en áreas rurales que según Freeman todavía son considerados por los investigadores (Loomis, 1946; Loomis \& Davidson, 1939; Loomis \& Powell, 1949).

\subsection{Los años cincuenta (Capítulo 6)}

Durante los cincuenta en la Universidad de Lund (Suecia) hubo importantes contribuciones bajo un enfoque estructural por parte del geógrafo Torsten Hägerstrand. Hägerstrand y su futura esposa realizaron un estudio individual de los pobladores de Asby entre 1840 y 1940, llegando a recolectar más de 10 mil patrones de migración. Hägerstrand no se conformada con la recolección descriptiva de los datos y, bajo la influencia de otros autores como Arthur Eddington, George Lundberg, A. J. Lotka, Moreno y Lewin, aplicó el enfoque estructural a sus estudios sobre la difusión de innovaciones. Hägerstrand, por medio de la simulación computacional pudo demostrar, a través de una secuencia de mapas, la difusión de innovaciones en el tiempo. De esta manera adaptó el modelo Montecarlo (Hägerstrand, 1965), presentado por Carl-Erik Fröberg con escaso éxito, a una simulación de difusión aleatoria persona a persona, asumiendo que había una probabilidad más alta de una mayor difusión de información entre personas cercanas que lejanas. Estas simulaciones se expresaban por medio de ciclos que iban pasando de un actor a otro (Hägerstrand, 1952).

Paralelamente, durante los años cincuenta en la Universidad de Chicago se realizaron estudios estructurales en el Comité de Biofísica Matemática, donde se desarrollaron aplicaciones matemáticas para resolver problemas sociológicos. Entre los integrantes del comité se encontraban, entre otros, Anatol Rapoport y Hyman G. Landau, los cuales trabajaron modelos de jerarquía (Landau \& Rapoport, 1953). Estos investigadores tuvieron gran reconocimiento, pero el más destacado fue el matemático Rapoport.

Entretanto, Paul Lazarsfeld y Robert K, Merton en la Universidad de Columbia a mediados de los cincuenta desarrollaron un enfoque de análisis de redes sociales (Lazarsfeld \& Merton, 1954). Lazarsfeld comenzó estudiando el comportamiento del consumidor y el impacto del desempleo para más tarde estudiar el impacto de la radiodifusión en el comportamiento. Lazarsfeld y Merton trabajaron de manera conjunta y colaboraron en muchos proyectos sobre comunicación y sobre la amistad (Lazarsfeld \& Merton, 1954). Freeman destaca, sin embargo, que la mayor contribución que hicieron es formar estudiantes como Coleman, Menzel y Katz, que estudiaron los factores sobre la difusión de información entre médicos (Coleman, Katz, \& Menzel, 1957). Según apunta Freeman, Lazarsfeld había trabajado con Moreno y Jennings y había recibido la influencia de estos.

En la Universidad de Iowa, a mediados de los años cincuenta, Everett Rogers, un estudiante de sociología aplicó técnicas sociométricas a la difusión de innovaciones. Rogers había leído a Moreno e influenciado bajo sus ideas, realizó 155 encuestas a granjeros en Iowa. De esta manera, Rogers estudió los patrones de difusión de información utilizando un enfoque sociométrico en el tiempo, y consiguió aplicar un enfoque estructural para analizar la adopción y difusión de innovaciones. Más tarde se vio influenciado por Lazarsfeld y el grupo de Columbia, y realizó análisis estructurales más complejos. Tras su paso como profesor por la Universidad Estatal de Ohio fue a 
trabajar al Departamento de las Ciencias de la Comunicación en la Universidad de Michigan. Su trabajo continuó y formó alumnos importantes en el análisis de redes sociales y realizó importantes contribuciones (Rogers, 1962).

Por otra parte, en Gran Bretaña, tanto Bronislaw Kaspar Malinowski como Alfred Reginald Radcliffe-Brown trabajaron con un enfoque estructural, siendo RadcliffeBrown o R-B conocido por la aplicación de dicho enfoque, defendiendo en muchas ocasiones el desarrollo de herramientas para el estudio (Radcliffe-Brown, 1940).

Paralelamente, en la Universidad de Syracuse, tanto Morris H. Sunshine como el propio Linton Freeman impartieron clases. Según describe Freeman, gracias a Warner Bloomberg Jr. obtuvieron el apoyo de la Fundación Ford para realizar estudios sobre la toma de decisiones comunitarias en Syracuse. Freeman nació en Chicago en 1927 y recibió las influencias del pensamiento estructural desde diversas fuentes como, por ejemplo, las publicaciones de Hägerstrand sobre la difusión de innovaciones y los estudios de Bavelas y Leavitt en el MIT, entre otros (Bavelas, 1948; Hägerstrand, 1952; Hägerstrand, 1965; Leavitt, 1951). Finalmente, según apunta el autor, quedó impresionado con un artículo de Rapoport y animó a alguno de sus estudiantes a realizar una tesis bajo el modelo de Rapoport. Más tarde, Freeman comenzó a trabajar con Morry Sunshine y otros investigadores sobre la toma de decisiones comunitarias desde una perspectiva estructural. A finales de los sesenta Freeman leyó un artículo "revelador" de Rapoport y Horvath (1961) donde pudo ver que trabajos como el de Davis, Gardner y Gardner, Hägerstrand, Bavelas y Leavitt, Bott y Rapoport tenían el rasgo en común de estar todos enfocados en la interacción entre actores sociales bajo un enfoque estructural (Bavelas, 1948; Bott, 1928; Davis et al., 1941; Leavitt, 1951; Rapoport \& Horvath, 1961).

\subsection{Los años sesenta (Capítulo 7)}

Los cuatro grupos de investigación que continuaron con el análisis de redes desde una perspectiva estructural se encontraban en el Laboratorio de Psicología Experimental de la Sorbona en París a principios de los sesentas, la Universidad de Michigan, la Universidad de Chicago y la Universidad de Ámsterdam.

En primer lugar, en la Sorbona, el psicólogo Claude Flament trabajó en el mencionado laboratorio de piscología junto con otros científicos. Flament conocía las investigaciones de Bavelas y Leavitt de los años 1956 y 1951, respectivamente, y las investigaciones de Cartwright y Harary de 1956 y la teoría de gráficos de Harary y Norman (1953). En 1961, Flament, que había trabajado anteriormente con gráficas, fue invitado a participar en un programa de verano organizado por el reconocido Lazarsfeld. James Coleman, editor de la colección Análisis Matemático y Comportamiento pidió a Flament que contribuyera con un libro. En 1963 se publicó el libro Teoría de Gráficas y Estructura de Grupos donde se contempla información estructural e imágenes gráficas (Flament, 1963). El trabajo de Flament todavía es considerado como uno de los importantes dentro del análisis de redes sociales.

En segundo lugar, a mediados de los sesenta, en la Universidad de Michigan, Edward Laumann estaba interesado en el análisis de redes sociales gracias a las influencias de Harrison White. Laumann estudió problemas de estratificación, política y comportamiento sexual, bajo la influencia del enfoque estructural de Warner (Laumann, 1973, 2000). El papel de Laumann es destacado debido a que formó estudiantes en la Universidad de Michigan, que más tarde se han convertido en notables investigadores en este campo. 
En tercer lugar, hacia finales de los sesentas en la Universidad de Chicago, Peter Blau y James A. Davis trabajaron con enfoques estructurales (Blau \& Duncan, 1967; Davis \& Leinhardt, 1967). Peter Blau tuvo influencias de George Simmel, Talcott Parsons, Robert Merton y Paul Lazarsfeld y gracias a esto adoptó en sus investigaciones un enfoque estructural. Davis por su parte, estuvo interesado en la teoría de gráficas. Tanto Blau como Davis aplicaron un enfoque de redes realizando importantes aportes.

Paralelamente, a finales de los sesentas, en la Universidad de Ámsterdam se trabajaba con enfoques estructurales y tuvo un importante impacto entre los sociólogos europeos. Robert Mokken, especialista en estadística estudió los problemas de comunicación de masas y conocía estudios como los de Katz y Lazarsfeld (1966) y Everett Rogers (1962) entre otros.

\section{CONSOLIDACIÓN DEL ANÁLISIS DE REDES (CAPÍTULO 8)}

La Universidad de Harvard tuvo un papel destacado para él análisis de redes sociales. En 1959 James Coleman fundó un departamento en la Universidad Johns Hopkins y contrataron a Harrison White de la Universidad de Chicago. White, formado en física teórica, tenía el conocimiento suficiente como para aplicar los fundamentos matemáticos al análisis estructural. White comenzó a trabajar en la Universidad de Harvard en el 1963 realizando interesantes aportes formando a toda una generación de estudiantes bajo el enfoque estructural. De esta manera, según describe Freeman a través Abbott (1994): "White es un hombre que inició revoluciones sociológicas, introdujo nuevas técnicas, y formó a los mejores grupos de estudiantes". Entre tanto, algunos de los trabajos más notables en el análisis de redes son los realizados por White y sus estudiantes (White, 1963; White, Boorman, \& Breiger, 1976).

Por otra parte, en este mismo capítulo "El análisis de redes se organiza", se explora con la habilidad que caracteriza a Linton Freeman, la fragmentación de las distintas escuelas e investigadores influyentes a través de un enfoque estructural. El resultado muestra que los sociólogos estaban mucho más desconectados entre ellos mismos que entre investigadores de otras disciplinas.

Continuando con este capítulo, hay algunos aspectos a destacar que han tenido un papel importante en el análisis de redes sociales entre los cuales están: 1) los programas computacionales; 2) las conferencias; 3) el INSNA; 4) la revista Social Networks; 5) el EISES; 6) los "Sunbelt" y; 7) el papel de la Universidad de Irvine.

1) Los programas de computación en el análisis de las redes sociales han marcado un antes y un después. Según Freeman, los primeros programas desarrollados eran para recoger información interpersonal, como el desarrollado por James Coleman y Duncan MacRae en 1960. Más tarde se desarrollarían diversos programas con el fin del ir resolviendo problemas. Algunos de ellos son SOCPAC 1 (Leinhardt, 1971), BLOCKER (Heil \& White, 1976), SOCK (Alba \& Gutmann, 1972), entre otros. Durante los ochentas aparecieron programas destacables como el conocido UCINET (Borgatti, Everett, \& Freeman, 2002).

2) Las conferencias desarrolladas bajo el enfoque de análisis de redes acogieron a investigadores de distintas universidades generando redes de conocimiento. Entre las primeras se encuentra una serie de cuatro conferencias organizadas por el geógrafo 
Forrest R. Pitts de la Universidad de Hawaii. Los eventos lograron acoger a investigadores como Everett M. Rogers y Lawrence Kincaid.

3) Otra de las contribuciones importantes fue la de Barry Wellman a través la Red Internacional de Análisis de Redes Sociales o "Internacional Network for Social Analysis", por sus siglas en inglés "INSNA", donde se creó una lista de miembros del consejo en la cual estaba constituida por estudiantes de diversas disciplinas, como ciencias de la computación, economía, educación, matemáticas, sociología y geografía entre otras.

4) Las revistas también tuvieron un lugar destacado. La revista Social Networks nace de la idea de Linton Freeman de disponer de una organización de las publicaciones científicas. Su propuesta de proyecto fue aceptada por las editoriales y en 1978 se publicó el primer número en Social Networks. Según señala Freeman (p. 154): "la revista ha jugado un papel unificador muy importante entre los practicantes del análisis de redes sociales".

5) El proyecto Sistema Electrónico de Intercambio de Información, por sus siglas en inglés "EIES", era algo similar a una comunicación de mensajería por internet, que pretendía poner en contacto a científicos remplazando el cara a cara. En este proyecto experimental se esperaba analizar si la comunicación a través de la computadora podía mejorar la eficiencia de los científicos.

6) Los "Sunbelt" son congresos anuales internacionales que pretendieron integrar a la comunidad de investigadores en análisis de redes sociales. Comenzaron en EEUU en 1981 y estuvieron organizados por H Russell Bernard y Alvin W. Wolfe. Más tarde los congresos saltaron a Europa, y en la actualidad se siguen celebrando con gran participación.

7) Finalmente, la Universidad de California en Irvine (UCI), ha tenido un distinguido papel en el análisis de redes sociales. Freeman fue nombrado decano de la escuela de Ciencias Sociales de dicha universidad en el 1979. Durante este periodo, Freeman junto con otros investigadores, trabajaron en un proyecto de investigación de redes sociales. Este proyecto sirvió para poner en marcha un programa de doctorado en análisis de redes sociales que atrajo multitud de estudiantes y profesores.

\section{CONCLUSIÓN}

El libro "El desarrollo del análisis de redes sociales: un estudio de sociología de la ciencia", es un libro que se lee de manera sencilla y que es útil para conocer la evolución que ha tenido el análisis de redes sociales en la historia. Debido a la fecha de la publicación del título original en inglés en el año 2004, y la posterior publicación de la versión traducida al español en el año 2012 (Freeman, 2012), se pueden echar en falta algunos datos más recientes para culminar con un libro que pudiera haber sido una buena referencia en la historia del análisis de redes sociales. Sin duda, en el momento de la publicación, en el año 2004, se trataría de un ejemplar de referencia, sobre todo, en lo que respecta a los capítulos finales "El análisis de redes se organiza" y "Un sumario y varias sorpresas", capítulo 9 y 10 respectivamente, que son los que contendrían la información más actualizada del análisis bibliográfico. Sin embargo, a excepción de estos capítulos y quizá algunos matices puntuales, el resto del libro, que trata sobre la evolución en el tiempo del análisis de redes hubiera seguido apenas sin cambios. Es importante también 
destacar que la obra no se corresponde con un manual teórico-práctico de análisis de redes sociales tan útiles para los investigadores, sino que, a diferencia de aquellos, este libro se enfoca en la parte humana de la construcción de la ciencia, y deja entrever las redes sociales entre investigadores que se han erigido, en buena medida, entre disciplinas separadas que finalmente convergieron para dar lugar a lo que a día de hoy conocemos como el análisis de redes sociales. Por otro lado, Freeman, a través una profunda revisión bibliográfica pone en el centro de la disciplina de análisis a multitud de estudiantes e investigadores, abarcando un amplio periodo en declive durante el oscurantismo, pero con una explosión intelectual en Harvard. Así mismo, el autor apunta hacia diversas figuras intelectuales tales como Harrison White o Jacob Moreno como personajes influyentes y reconocidos en la disciplina de redes para muchos investigadores, sin olvidar la figura de Helen Jennings, mucho menos reconocida en esta disciplina y que tuvo una gran influencia en los trabajos de Moreno.

Finalmente, no cabe duda que Linton Freeman realiza un profundo trabajo sobre el desarrollo del análisis de redes sociales contribuye a enriquecer el entendimiento de la disciplina desde el punto de visto histórico. Sin embargo, muchos investigadores que estén adentrándose en el mundo de las redes sociales no encontrarán en este libro distinciones entre contribuciones sociocéntricas y egocéntricas, las cuales serían de utilidad con el fin de conocer la evolución histórica de dichos enfoques y tener al alcance referencias bibliográficas diferenciadas.

\section{REFERENCIAS}

Abbott, A. (1994). Identity and Control: A Structural Theory of Social Action.By Harrison C. White. Princeton University Press. Social Forces, 72(3), 895-901. https://doi.org/10.1093/sf/72.3.895

Alba, R. D., \& Gutmann, M. P. (1972). SOCK: A Sociometric Analysis System. SIGSOC Bull, 3(3), 11-12. https://doi.org/10.1145/1103251.1103252

Almack, J. C. (1922). The influence of intelligence on the selection of associates. School and Society, 16(410), 529-530.

Bavelas, A. (1948). A mathematical model for group structures. Applied Anthropology, 7(3), 16-30.

Blau, P. M., \& Duncan, O. D. (1967). The American occupational structure.

Borgatti, S. P., Everett, M. G., \& Freeman, L. C. (2002). Ucinet for Windows: Software for social network analysis. Harvard, MA: Analytic Technologies, 2006.

Bott, H. (1928). Observation of play activities in a nursery school. Genetic Psychology Monographs, 4(1), 44-88.

Chapple, E. D., \& Arensberg, C. M. (1940). Measuring human relations: an introduction to the study of the interaction of individuals. The Journal Press.

Coleman, J., Katz, E., \& Menzel, H. (1957). The diffusion of an innovation among physicians. Sociometry, 20(4), 253-270.

Coleman, J. S., \& MacRae, D. (1960). Electronic processing of sociometric data for groups up to 1.000 in size. American Sociological Review, 25(5), 722-727.

Comte, A., \& Martineau, H. (1853). The positive philosophy of Auguste Comte. D. 
Appleton.

Davis, A., Gardner, B. B., Gardner, M. R., \& Warner, W. L. (1941). Deep South; a social anthropological study of caste and class. The University of Chicago Press.

Davis, J. A., \& Leinhardt, S. (1967). The structure of positive interpersonal relations in small groups.

Flament, C. (1963). Applications of graph theory to group structure. Prentice-Hall.

Freeman, L. C. (1978). Centrality in social networks conceptual clarification. Social Networks, $\quad 1(3), \quad 215-239 . \quad$ https://doi.org/https://doi.org/10.1016/03788733(78)90021-7

Freeman, L. C. (2004). The Development of Social Network Analysis: A Study in the Sociology of Science. Retrieved from https://books.google.es/books?id=VcxqQgAACAAJ

Freeman, L. C. (2012). El Desarrollo del Análisis de Redes Sociales: Un estudio de Sociología de la Ciencia. Palibrio.

Hägerstrand, T. (1952). The propagation of innovation waves. London: Royal University of Lund.

Hägerstrand, T. (1965). A Monte Carlo approach to diffusion. European Journal of Sociology/Archives Européennes de Sociologie, 6(1), 43-67.

Hagman, E. P., \& Stoddard, G. D. (1933). The Companionships of Preschool Children ... Retrieved from https://books.google.es/books?id=aCDBtwAACAAJ

Harary, F., \& Norman, R. Z. (1953). Graph theory as a mathematical model in social science. University of Michigan, Institute for Social Research Ann Arbor.

Heil, G. H., \& White, H. C. (1976). An algorithm for finding simultaneous homomorphic correspondences between graphs and their image graphs. Behavioral Science, 21(1), 26-35. https://doi.org/10.1002/bs.3830210105

Hobson, J. A. (1894). The Evolution of Modern Capitalism: A Study of Machine Production. Retrieved from https://books.google.es/books?id=Vlc1AQAAMAAJ

Homans, G. C. (2013). The human group. Routledge.

Huber. (1810). Recherches sur les mours des fourmis indigènes. Retrieved from https://books.google.fr/books?id=185iAAAAcAAJ

Huber, \& Bonnet, C. (1792). Nouvelles observations sur les abeilles: adressées à M. Charles Bonnet. Retrieved from https://books.google.es/books?id=L3dlAAAAMAAJ

Katz, E., \& Lazarsfeld, P. F. (1966). Personal Influence, The part played by people in the flow of mass communications. Transaction publishers.

Landau, H. G., \& Rapoport, A. (1953). Contribution to the mathematical theory of contagion and spread of information: I. Spread through a thoroughly mixed population. The Bulletin of Mathematical Biophysics, 15(2), 173-183.

Laumann, E. O. (1973). Bonds of pluralism: The form and substance of urban social networks. J. Wiley.

Laumann, E. O. (2000). The social organization of sexuality: Sexual practices in the United States. University of Chicago press. 
Lazarsfeld, P. F., \& Merton, R. K. (1954). Friendship as a social process: A substantive and methodological analysis. Freedom and Control in Modern Society, 18(1), 1866.

Leavitt, H. J. (1951). Some effects of certain communication patterns on group performance. The Journal of Abnormal and Social Psychology, 46(1), 38.

LeBon, G. (1895). The crowd. London: F. Unwin.

Leinhardt, S. (1971). SOCPAC I : A FORTRAN IV program for structural analysis of sociometric data. Behavioral Science, 16, 515-516.

Loomis, C. P. (1946). Political and Occupational Cleavages in a Hanoverian Village, Germany: A Sociometric Study. Sociometry, 9(4), 316-333. https://doi.org/10.2307/2785496

Loomis, C. P., \& Davidson, D. (1939). Sociometrics and the Study of New Rural Communities. Sociometry, 2(1), 56-76. https://doi.org/10.2307/2785398

Loomis, C. P., \& Powell, R. M. (1949). Sociometric Analysis of Class Status in Rural Costa Rica-A Peasant Community Compared with an Hacienda Community. Sociometry, 12(1/3), 144-157. https://doi.org/10.2307/2785382

Luce, R. D., \& Perry, A. D. (1949). A method of matrix analysis of group structure. Psychometrika, 14(2), 95-116. https://doi.org/10.1007/BF02289146

Macfarlane, A. (1883). Analysis of relationships of consanguinity and affinity. The Journal of the Anthropological Institute of Great Britain and Ireland, 12, 46-63.

Marineau, R. (1989). Jacob Levy Moreno, 1889-1974: Father of Psychodrama, Sociometry, and Group Psychotherapy. Retrieved from https://books.google.co.cr/books?id=O0oOAAAAQAAJ

Moreno. (1937). Sociometry in Relation to Other Social Sciences. Sociometry, 1(1/2), 206-219. https://doi.org/10.2307/2785266

Moreno. (1953). Who shall survive? Foundations of sociometry, group psychotherapy and socio-drama, 2nd ed. In Who shall survive? Foundations of sociometry, group psychotherapy and socio-drama. Beacon House.

Moreno, J. L., \& Jennings, H. H. (1938). Statistics of social configurations. Sociometry, 342-374.

Moreno, \& Jennings, H. H. (1960). The Sociometry Reader. Edited by J.L. Moreno, with Helen H. Jennings [and Others], Etc. Retrieved from https://books.google.es/books?id=wHwqMwEACAAJ

Morgan, L. H. (2018). League of the Ho-dé-no-sau-nee, or, Iroquois. Retrieved from http://online.canadiana.ca/view/oocihm.38467

Radcliffe-Brown, A. R. (1940). On social structure. The Journal of the Royal Anthropological Institute of Great Britain and Ireland, 70(1), 1-12.

Rapoport, A., \& Horvath, W. J. (1961). A study of a large sociogram. Behavioral Science, 6(4), 279-291.

Roethlisberger, F. J., \& Dickson, W. J. (1939). Dickson. WJ (1939). Management and the worker. Cambridge, MA.

Rogers, E. M. (1962). Diffusion of innovations. Prensa Libre. 
Simmel, G., \& Levine, D. N. (1971). On individuality and socialforms: selected writings.

Warner, W. L. (1975). Yankee City. Yale University Press.

Warner, W. L., \& Lunt, P. S. (1941). The social life of a modern community.

Wellman, B. (1926). The school child's choice of companions. The Journal of Educational Research, 14, 126-132. https://doi.org/10.1080/00220671.1926.10879675

White, H. C. (1963). An Anatomy of Kinship: Mathematical Models for Structures of Cumulated Roles. Retrieved from https://books.google.es/books?id=WogDAAAAMAAJ

White, H. C., Boorman, S. A., \& Breiger, R. L. (1976). Social structure from multiple networks. I. Blockmodels of roles and positions. American Journal of Sociology, 81(4), 730-780.

Rafael Mesa Manzano

Graduado en Geografía y Medio Ambiente

Máster en Técnicas para la Gestión del Medio Ambiente y del Territorio

Universidad de Valencia 